\title{
A Modified Relativistic Model of Gravitational Propagation
}

\author{
Jonathan Peter Merrison \\ Institute of Physics and Astronomy, Aarhus University, Aarhus, Denmark \\ Email: merrison@phys.au.dk
}

Received 29 July 2016; accepted 17 September 2016; published 21 September 2016

Copyright (C 2016 by author and Scientific Research Publishing Inc.

This work is licensed under the Creative Commons Attribution International License (CC BY). http://creativecommons.org/licenses/by/4.0/

(c) (i) Open Access

\section{Abstract}

A novel model of gravity is proposed and developed by modifying general relativity through propagating the gravitational field in an entirely analogous way to that of electromagnetic fields. It is therefore not a purely geometric model of gravitation, but is self-consistent, having clear causality and has the benefit of being inherently compatible with unified field theories. This model reproduces the observed almost constant rotational velocities of many galaxies as well as other large scale non-Keplerian motion. This is achieved without assuming the existence of dark matter and is made possible by modelling a rapidly rotating central star which with the inclusion of a velocity induced Doppler shift (of gravity) generates a highly anisotropic and intense, sheet like gravitational field. At extremely high gravitational fields this model remains real and finite i.e. does not generate a black hole, instead it asymptotically approaches a field limit below which light may escape. This is due to the inclusion of self-interaction of gravity in vacuum leading to a non-linearity in the propagation of gravitational energy i.e. the effects of a gravitational field upon itself. This model is implemented computationally using an iterative finite element model. On the scale of our solar system these corrections are small and are shown not to be in obvious disagreement with high precision solar system tests.

\section{Keywords}

Galaxy Rotation, Gravitation, General Relativity

\section{Introduction}

Despite the success of general relativity in high precision experiments within the solar system there are many extremely troubling features of this theory when applied to larger spatial and mass scales. Specifically the failure to reproduce observed motion within and between galaxies. The occurrence of infinities is also normally taken as a sign of unphysical behavior (i.e. the prediction of black holes), with associated problems of causality and 
paradoxes. Additionally attempts to integrate general relativity into unified theories have failed. This has stimulated the proposal of theories competing with or modifying general relativity.

One of the most important observational features of galaxies (spiral, elliptical, lenticular, etc.) is their approximately constant rotational velocity which does not follow expected Keplerian motion $(v \propto 1 / \sqrt{r})$,almost as if gravitational acceleration had a $1 / r$ dependence within the galactic disc (e.g. [1] [2]). This has been formalized in the Tully-Fisher law where the radial acceleration in a galaxy is seen to follow; $a=-G \sqrt{M} / r$ [3]-[5]. Non-Keplerain motion is also observed in many large scale observations of gravity, for example velocities of galaxies in clusters, the orbits of wide binary stars [6]-[8] and in gravitational lensing [5] [9] [10]. It has been a challenge to modern gravitational theory to explain these observations. The conventional proposal of complex distributions of large amounts of invisible "cold dark matter" (CDM) model [11] [12] has also been controversial and has led to competing gravitational theories to be sought. The most popular of such models is the MOND (Modified Newtonian Dyanamics) [13]-[18] in which Newtonian laws are modified at large scale/weak fields for galaxies. Another model modifies general relativity i.e. the modified gravitational theory (MOG) or scalar-tensor-vector gravity (STVG) [19] [20]. Although these models have not been widely accepted this issue remains actively debated, it is still a challenge to model gravity at large scale [21]. In the work presented here although some of the basic assumptions employed in general relativity are questioned, especially at high field and high velocities, the model proposed here is founded upon relativity theory and should be seen as a 'modification' of general relativity as applied to gravitation.

In general relativity gravitational effects are modelled as a set of accelerated (accelerating) frames of reference and thereby as curvature of space time. It employs not just Galilean or weak equivalence, but furthermore assumes, "the complete physical equivalence of a gravitational field and a corresponding acceleration of the reference system”. i.e. accelerating frames are indistinguishable from rest frames. The basic components of general relativity are therefore modelling a gravitational field by the transport (in an accelerated frame) of electromagnetic radiation also including the effect of the gravitational field (curved space-time) upon the generation of gravitation. It is in this way non-linear (including the gravitational energy in the total energy-momentum tensor), however the gravitational field is propagated independently of the gravitational field. In general relativity it is assumed that; "The space-time geometry is entirely determined by the energy-momentum tensor of matter" i.e. it employs strong equivalence in a metric theory of gravity [22].

In the work presented here a gravitational field is assumed to propagate in an entirely analogous fashion to that of an electro-magnetic field. This imposes an additional non-linearity, not currently present in general relativity, which corresponds to the (vacuum) interaction of a gravitational field upon itself. This will be especially significant at high gravitational fields (g) and long distance (r) i.e. $g r \rightarrow c^{2}$. Similarly the propagation of gravitational fields will also be susceptible to relative velocity of the emitting frame i.e. Doppler effects, these again are not present in general relativity and relative motion, only appearing in an indirect sense through variation of energy-momentum and thus the generation of the gravitational field. Clearly this relative velocity induced effect would become significant at high relative velocities of a gravitating body.

In this paper these modifications are quantified for a few physically significant cases. In this paper a simple finite element computational approach is taken to quantify the effect of gravitational propagation induced non-linearity (section 2) applicable in the case of massive/compact (non-rotating) objects. The effects of high velocity (relativistic Doppler effects) on the propagation of gravitational fields will be crudely modelled in section 3 and applied to a rapidly rotating star at the galactic center in order to (quantitatively) describe the observed rotation curves of galaxies (such as spiral, elliptical, lenticular, etc.) including the Milky-way.

Since this model is founded upon relativity theory it is not in principle in disagreement with many precision predictions made under general relativity within the solar system (e.g. gravitationally induced time dilation). Deviations from general relativity caused by the proposed model will be discussed (quantified) in section 4 .

\section{Non-Linearity in Gravitational Field Propagation Applied to Massive/Dense Objects}

The aim here is to quantify the effect of including an additional non-linearity to the conventional general relativistic approach to gravitation. This non-linearity will be due to the effect of space time curvature (gravitational acceleration) upon the propagation of the gravitational field itself. In this work a computational approach was applied in which the problem was reduced to a discrete set of velocity transformations i.e. by integrating from 
each infinitesimally radially separated reference frame to the next.

In this computer model the gravitating object is assume to be spherically symmetric and modelled in (two dimensional) polar coordinates. The mass $\mathrm{M}$ and radius $\mathrm{R}$ of the object are variable, also the mass density is assumed to decrease with an adjustable scale height. The gravitational field is assumed to be emitted isotropically by each mass element within its emitting frame of reference. Integration is performed over all emitting angles and each radial mass element. Adjacent frames of reference (finite elements) are assumed to be held stationary with respect to each other and the gravitational field is propagated from each space-time frame to the next in an analogous manner to electromagnetic propagation, see Appendix 1.

By approximating the gravitational acceleration field to a discrete radial component of velocity difference; $d v \sim g_{0} \times d r / c$, allows the propagation between elements to be treated with a simple relativistic Doppler shift;

$$
g_{1}=g_{0} / \gamma(1-\beta \cos (\theta))
$$

where $g_{0}$ is the gravitational field as measured in the rest frame of a finite element (for example a mass containing emitting frame), $g_{1}$ is the magnitude of the gravitational field as measured in the receiving frame, the angle $(\theta)$ is that of the gravitational vector as measured in the receiving frame,

$$
\gamma=1 / \sqrt{\left(1-\frac{d v^{2}}{c^{2}}\right)}, \beta=\frac{d v}{c} .
$$

To be clear the $g_{0}$ determined here is the acceleration measured in a frame of reference held stationary with respect to both its surroundings and to a distant reference point. This choice has been made not just for simplicity, but also since the aim here is to quantify the propagation of gravity only. In a free-falling rest frame the gravitating mass within each element would be perceived to be accelerating radially outwards (with $g_{0}$ ) due to 'pressure' from the rest of the star i.e. forces preventing its collapse. Stating this in another way artificially holding these rest frames is in fact a realistic representation of the physical situation within a stable gravitating body i.e. the gravitating mass of a star/planet is not in free-fall.

This model employs the concept of 'gravitons' in the formal calculations, though it is important to stress that this should been seen as a generic form of gravitational theory with focus on gravitational propagation. The gravitons here should be seen only as representations of the transport of gravitational information (gravitational energy) in the same way that photons are employed in special relativity as a proxy for electromagnetic information transport. Specifically this model assumes that a volume of space-time containing gravitating mass $\mathrm{M}$ (energy-momentum) generates a spherically symmetric flux of 'gravitons'(as seen in its rest frame)which can be defined arbitrarily as a total rate of $4 \pi \mathrm{M}$ gravitons/s. The flux at a distance of $\mathrm{r}$ from this mass will be; flux $=$ $\mathrm{M} / \mathrm{r}^{2}$ gravitons $/ \mathrm{s} / \mathrm{m}^{2}$ in that rest frame. The gravitational acceleration (gravitational field strength) is defined as $g_{0}=G \times$ flux which for the weak field (non-relativistic case) becomes; $g_{0}=G M / r^{2}$ i.e. converging to Newtonian gravitation. The calculated gravitational field (element array) extends out to several orders of magnitude of the star radius. An iterative process is used to arrive at a self-consistent gravitational field, both within and outside the star, i.e. repeated propagation of the field (or gravitons)is performed until a self-consistent flux density field is achieved (within a definable accuracy which was typically $<30 \%$ ). At high fields more than 40 iterations were often necessary. Some of the benefits of using a computational model are its simplicity and transparency (eliminating the need for complex analytical solutions) combined with flexibility with regard to geometry, scale and accuracy. The element, time and angular resolution between infinitesimal rest frames can be adjusted such that convergence can be assured i.e. importantly $d v / c \ll 1$ and $g d r / c^{2} \ll 1$ etc.

In the results presented here a (much simplified) model of a sun-like star is used ( $\mathrm{M}=2 \times 10^{30} \mathrm{~kg}, \mathrm{R}=7 \times$ $10^{8} \mathrm{~m}$ ), it is assumed to be spherically symmetric and non-rotating. Figure $1(\mathrm{a})$ and Figure $1(\mathrm{c})$ show the computed gravitational acceleration as a function of radial distance, on two different scales, in the case of 1 solar mass within 1 solar radius $\left(6.9 \times 10^{8} \mathrm{~m}\right)$ under the Newtonian model (red) and the proposed model (blue). In this crude Newtonian calculation of gravity the mass is assumed to be a point source which probably accounts for the discrepancy seen within the star between the two models presented here. Figure 1(b) and Figure 1(d) show similar plots for the same 1 solar mass within a star radius of $3 \times 10^{-7}$ solar radii $(\mathrm{R}=207 \mathrm{~m})$. The vertical purple line shows the star surface. As can be seen the gravitational field is inhibited in the case of the more compact star. As shown in Figure 1(b) and Figure 1(d) at larger distance this non-linear model converges to a $\sim 1 / r^{2}$ dependence (though with an absolute reduction compared to the Newtonian model). 
In Figure 2(a) and Figure 2(b) the calculated gravitational acceleration g is plotted for various values of the mass (density), in Figure 2(a) the values are taken at the surface and in Figure 2(b) at a distance of around $10^{12}$ m. In Figure 3 the mass is held constant and the stars radius is reduced. In Figure 4 the product $g_{\text {surf }} R$ is plotted as a function of $\mathrm{M} / \mathrm{R}$, this allows easier comparison between models. The blue points and solid blue line are the computed data. The red line is the classical Newtonian calculated gravitational field. The vertical green dashed line in these figures indicates the Schwarzchild limit; $\left(R=2 G M / c^{2}\right)$, which is conventionally the mass and radius limit for the creation of an event horizon (i.e. where light cannot escape and a black hole is generated).

As can be seen in this model the gravitational field remains real and finite, asymptotically approaching the $g$ limit at which light propagation ceases. In Figure 4 the dashed red line indicates a crude, rough order of magnitude (ROM) estimate of this limit to the surface gravitational field;

$$
g_{\text {surf }} R<\sqrt{\frac{G M}{R}} \cdot \frac{c}{2}
$$

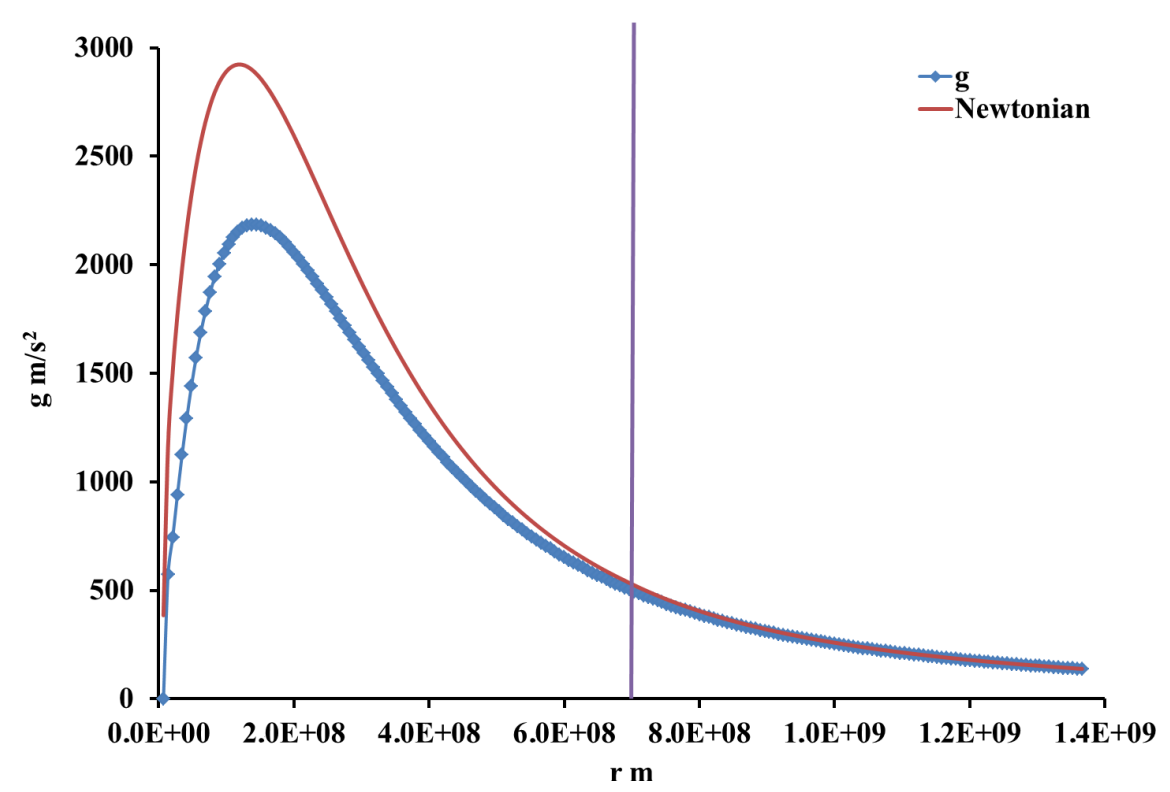

(a)

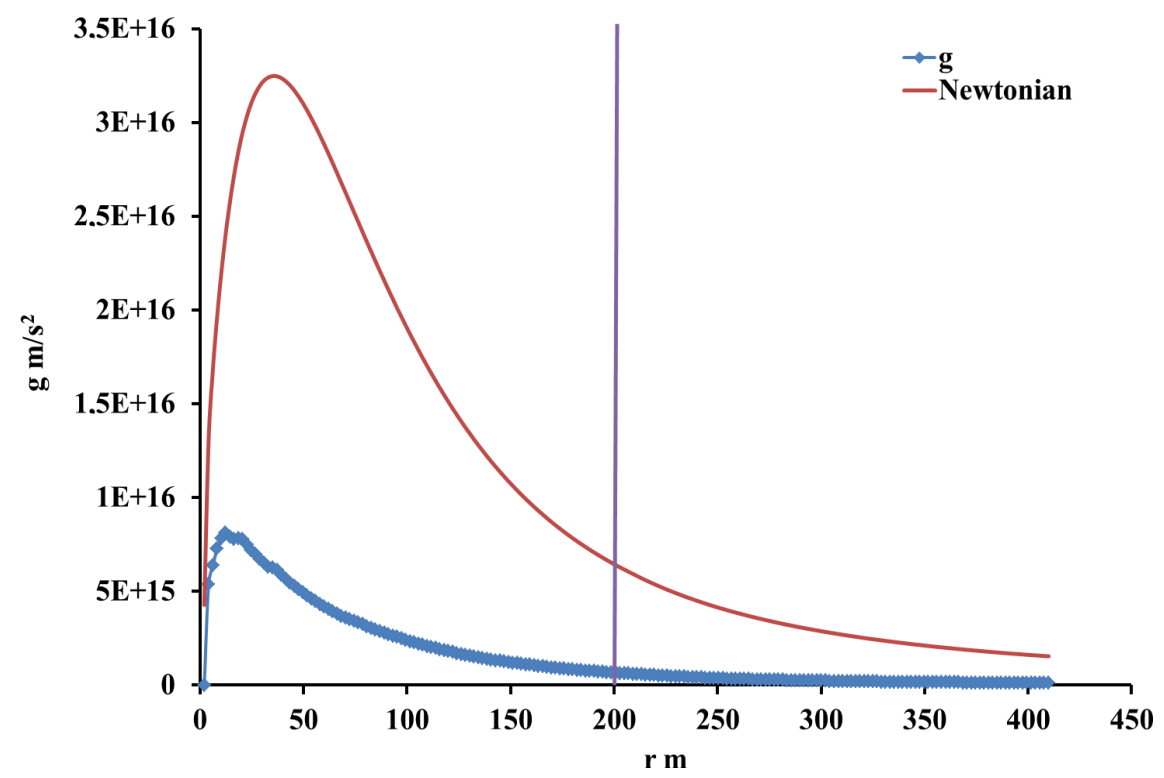

(b) 


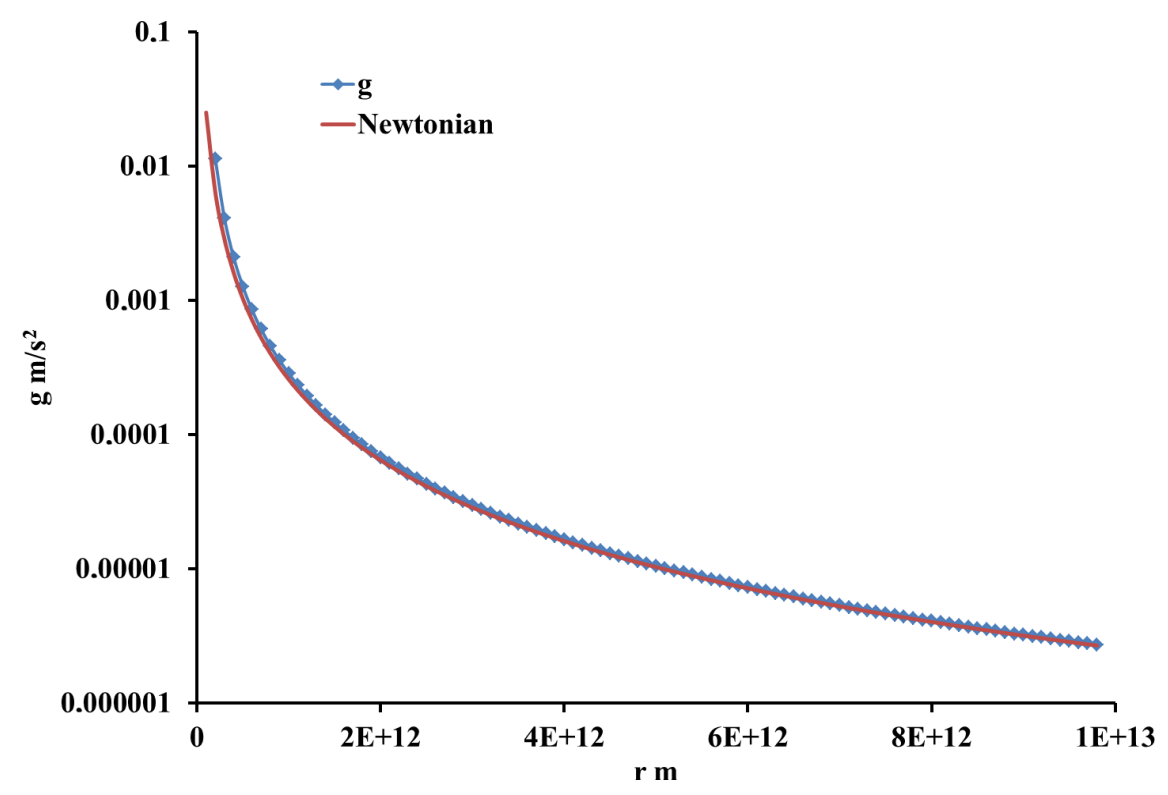

(c)

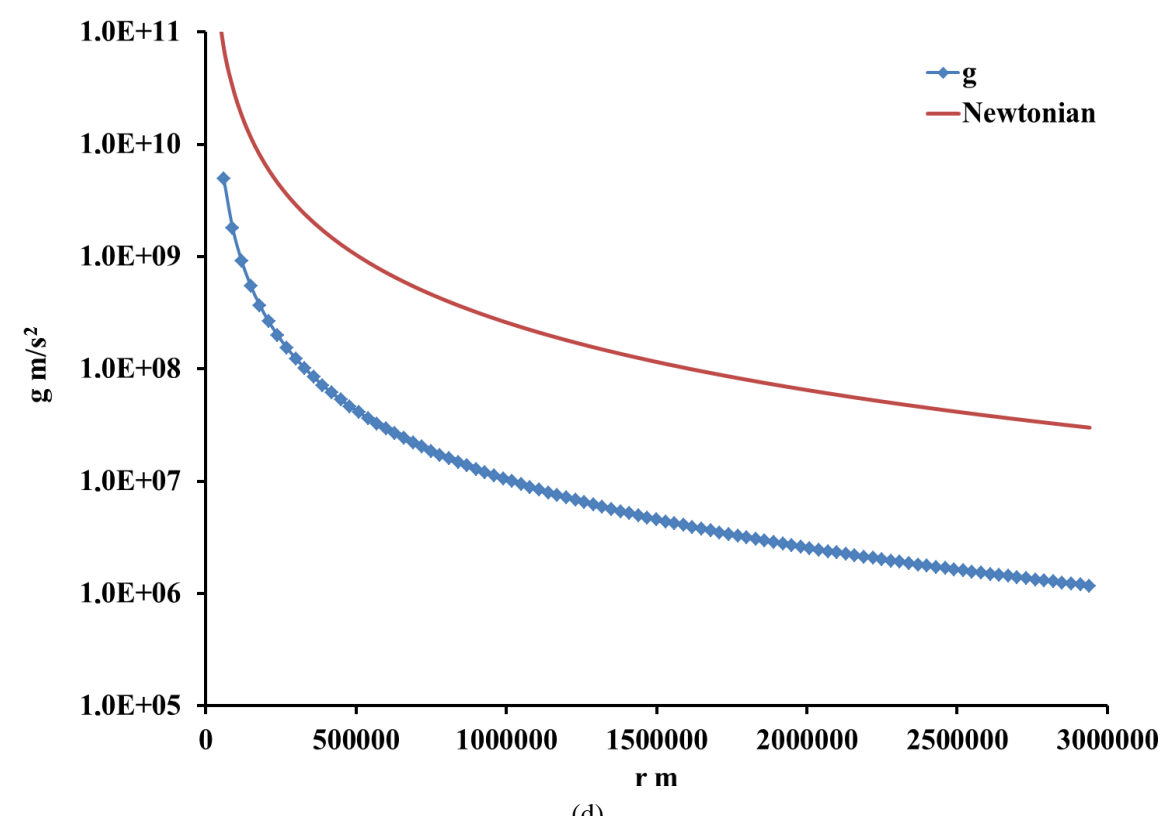

(d)

Figure 1. These plots show the calculated gravitational field strength as a function of distance from the center of a star of mass $\mathrm{M}=2 \mathrm{Msol}=3.9 \times 10^{30} \mathrm{~kg}$ and in (a) and (b) radius; $\mathrm{R}=\mathrm{Rsol}=6.9 \times 10^{8} \mathrm{~m}$, for (c) and (d) a radius of $\mathrm{R}=\mathrm{Rsol} \times 3 \times$ $10^{-7} \mathrm{~m}=207 \mathrm{~m}$. The blue line and dots are using this non-linear model and the red line is a simple Newtonian model. The vertical purple line shows the surface radius.

This limit is a direct result of the coupling of gravitation to itself during propagation i.e. the acceleration of gravity in the gravity field, of the form $\mathrm{d} g=-g \mathrm{~d} r / c^{2}$, giving an additional nonlinearity such that increasing the mass of an object will not linearly increase the observed gravitational field (or space-time curvature) and instead asymptotically approach this limit.

At extreme mass and compaction in this model there becomes essentially a limiting range (d) of the gravitational field ( $g d<c^{2}$ ) which may be thought of as an effective "penetration" depth of gravity due to its selfcoupling (non-linearity). This depth can be crudely estimated; $d \sim c / \sqrt{4 \rho G}$. This also leads to a limit of the surface gravitational acceleration which becomes only a function of the mass density and not individually the enclosed mass $(\mathrm{M})$ or size $(\mathrm{R})$ of the compact gravitating body i.e.; 


$$
g_{\text {surf }}<\sqrt{(\rho G)} c
$$

this crude approximation to the limit is shown in Figure 4 (dashed line).

\section{The Doppler Shift in Gravitational Field Propagation Applied to Rapidly Rotating Massive Stars and Galactic Rotation Curves}

An important and immediate consequence of the treatment of gravity as a propagating field (analogous to that of an electric field) as suggested in this paper is that the velocity of a gravitating mass will affect the propagated gravitational field. Relativistic effects will affect the gravitational field i.e. the gravitational field observed will

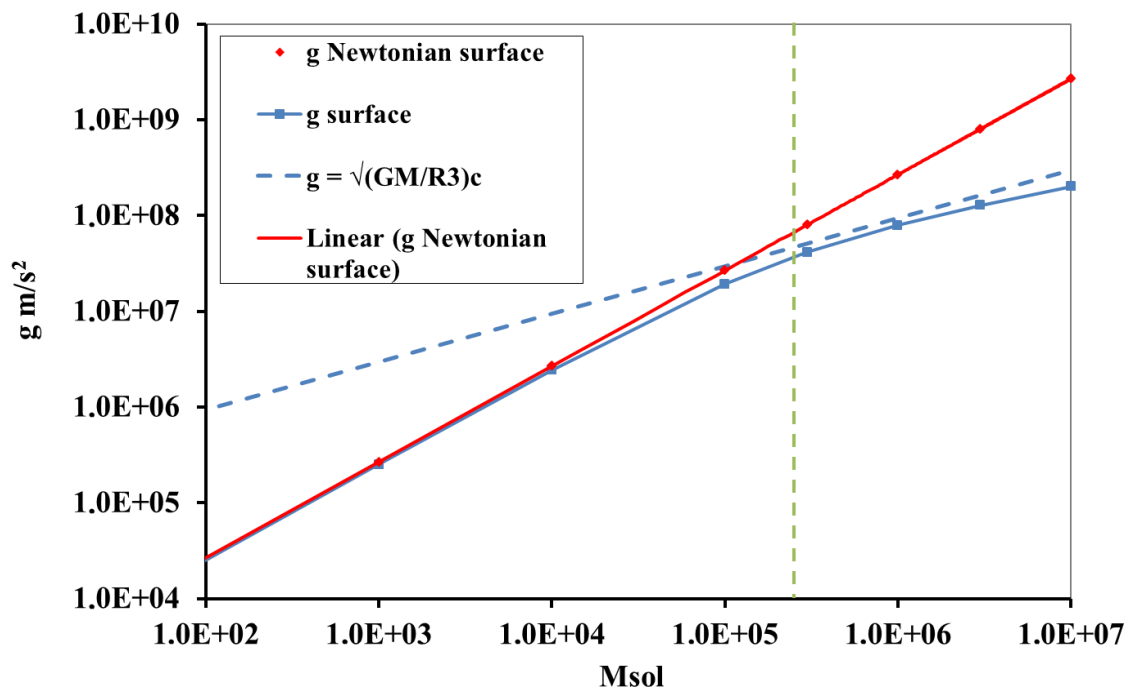

(a)

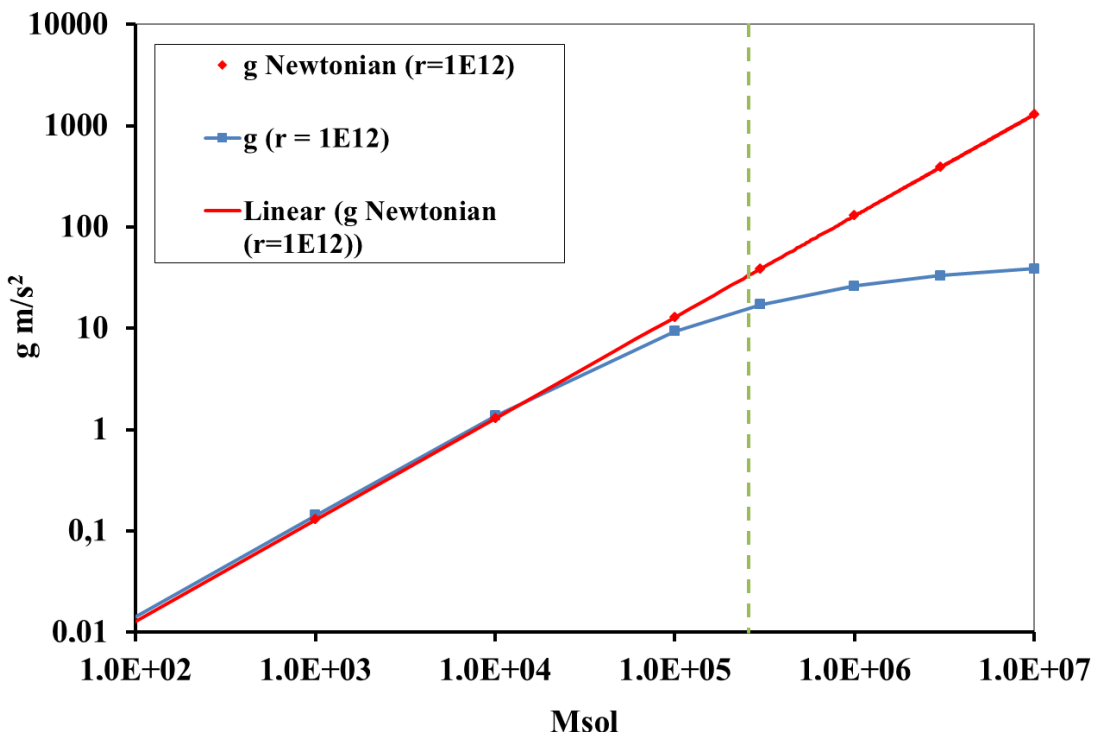

(b)

Figure 2. In these plots the gravitational field is quantified for a star of radius $\mathrm{R}=6.9 \times 10^{8} \mathrm{~m}=\mathrm{Rsol}$ and for different masses expressed in solar masses $1 \mathrm{Msol}=2 \times 10^{30} \mathrm{~kg}$. The red line (and points) are a simple Newtonian model. The vertical green dotted line corresponds to the mass corresponding to the Schwarzchild radius; $\mathrm{M}=\mathrm{Rc}^{2} / 2 \mathrm{G}$. The blue line and points are the current non-linear model. In (a) these calulations are at the surface of the star, in (b) at a radius of $1 \times 10^{12} \mathrm{~m}$. In Figure 2(a) the dotted blue line indicates a crude approximation to the limit of the surface gravitational field based on this non-linear model; $\mathrm{g}_{\text {surf }}<\sqrt{G M / R^{3}} \mathrm{c}$. 


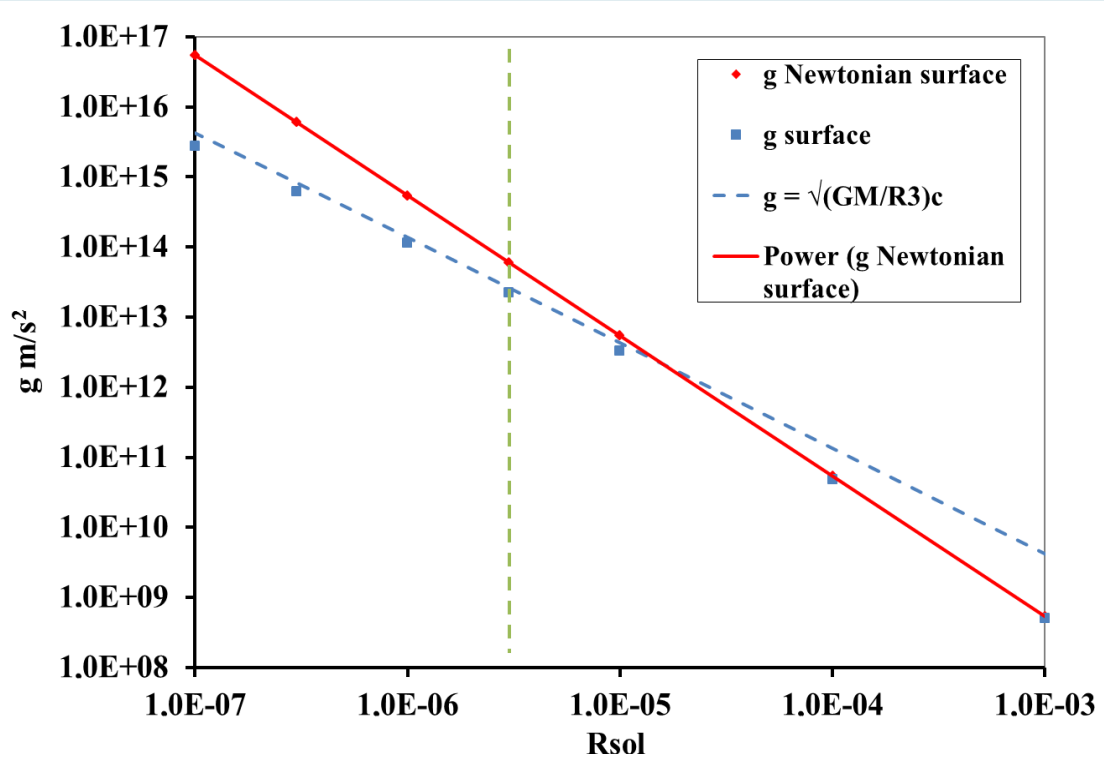

Figure 3. In this plot the gravitational field is quantified at the surface of a star of mass $\mathrm{M}=2 \times 10^{30} \mathrm{~kg}=1 \mathrm{Msol}$ and for different radii expressed in units of solar radius $\mathrm{R}=6.9 \times 10^{8} \mathrm{~m}=$ Rsol. The red line (and points) are a simple Newtonian model. The vertical green dotted line corresponds to the mass corresponding to the Schwarzchild radius; $\mathrm{R}=2 \mathrm{GM} / \mathrm{c}^{2}$. The blue points are the current non-linear model. The dotted blue line indicates a crude approximation to the limit of the surface gravitational field based on this non-linear model; $\mathrm{g}_{\text {surf }}<\sqrt{G M / R^{3}} \mathrm{C}$.

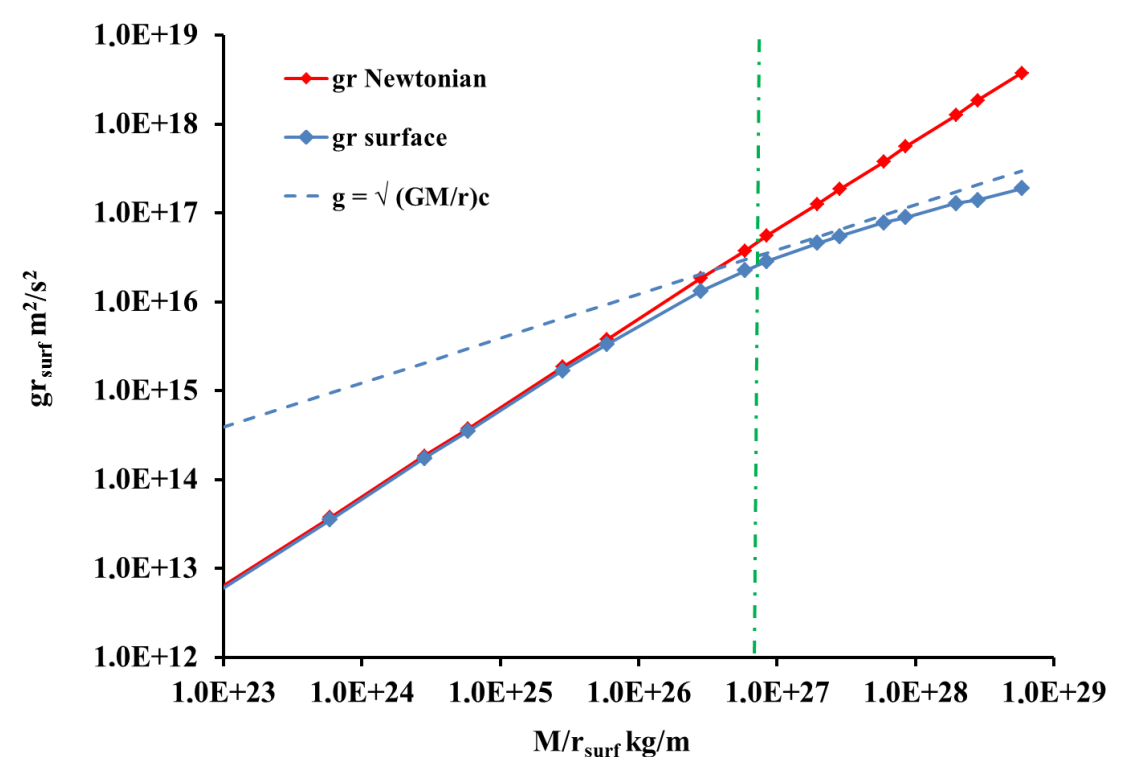

Figure 4. Plot of gr vs M/r (at the surface of the star) for different values of M (1 Msol to $1 \times 10^{7}$ Msol) and different values of $\mathrm{r}$ ( 1 Rsol to $1 \times 10^{-7}$ Rsol). General relativity (Scwarzchild limit) predicts that above $\mathrm{M} / \mathrm{r}=\mathrm{c}^{2} / 2 \mathrm{G}$ a black hole is created i.e. $\mathrm{M} / \mathrm{r}>7.7 \times 10^{26} \mathrm{~kg} / \mathrm{m}$ (green stippled line). The theory here predicts a limit to $g_{\text {surf }} R$ of around; $g_{\text {surf }} R<\sqrt{G M / R c} / 2$, shown with a dotted blue line.

be distorted at velocities approaching that of light. A gravitating body moving towards the observer will gravitate more (have an enhanced observed gravitational field) and one moving away will have a reduced gravitational pull i.e. one must apply the relativistic Doppler shift also to gravitational fields. This Doppler shift in gravity allows for the generation of highly anisotropic gravitational fields and thereby immediately begins to explain some of the structure and non-Keplerian motion observed on large scales and in most galaxies. The aim in this section is to quantify the effect of high rotation velocity on the propagation of the gravitational field gen- 
erated by a massive/compact star.

Ideally the effects of high velocity rotation upon the generation of a gravitational field would be (as in section 2) implemented into a finite element computational model with, for example, the addition of a relative velocity term (dv) between elements during integration. However this would involve additional dimensions and substantially increase integration (computational) times. Instead a simple model of a rotating star has been applied here in order to quantify the modification produced to the gravitational field of a rotating mass compared to a stationary mass. The rotation velocity is assumed to be constant and has been varied from a non-relativistic value ( $\mathrm{v}$ $\ll \mathrm{c}$ ) to a value as high as $\mathrm{v}=99.999 \%$ of c corresponding to $\gamma \sim 223$. The emitting angle to that of the rotation plane is varied from $-90^{\circ}$ to $+90^{\circ}$. Integration is made over all emitting angles within the plane of rotation. No consideration has been made here of the internal structure or stability of the star due to the simplicity of the model. The large relative velocity gradients expected within the star make it non-trivial to estimate its stability. However, the presence of intense magnetic fields could in principle readily provide necessary confinement of even weakly electrified plasma. Also the presence of internal slowly rotating mass could contribute additional gravitational confinement without detracting from the arguments presented here.

It is clear that at a high (relativistic) velocity each mass element of the object will generate essentially a beam of gravitational field which when integrated around the entire object will sweep out a sheet like emission of intense gravitation. Figure 5 shows the angular dependence of the emitted surface gravitational field at different relative rotation velocities assuming a star rest mass of $10^{5} \mathrm{Msol}$ and radius of $10^{12} \mathrm{~m}$. An angle of 0 degrees here is in the plane of rotation as observed by a stationary observer not rotating. Several orders of magnitude enhancement in $\mathrm{g}$ are seen within the rotation plane. Towards the axial direction there are several orders of magnitude reduction in $g$.

In Figure 6 a calculation is performed to show the expected rotation velocity curve for a galaxy containing a highly simplified massive central rapidly rotating star. As in Figure 5 the central star is assumed to have rest mass of $10^{5} M_{\text {sol }}$ and radius of $10^{12} \mathrm{~m}$. The galactic disc thickness is taken as $2 \mathrm{kpc}$. Within the disc the radial gravitational field as a function of radial distance is taken as the average over the disc thickness. This corresponds to an increasingly narrow angular range with distance from the star. It should be pointed out that in this model the variation of the gravitational field strength averaged over all angles is not strictly being modified from the conventional $1 / \mathrm{r}^{2}$ dependence. Rather that the angular dependence is being highly modified which may be

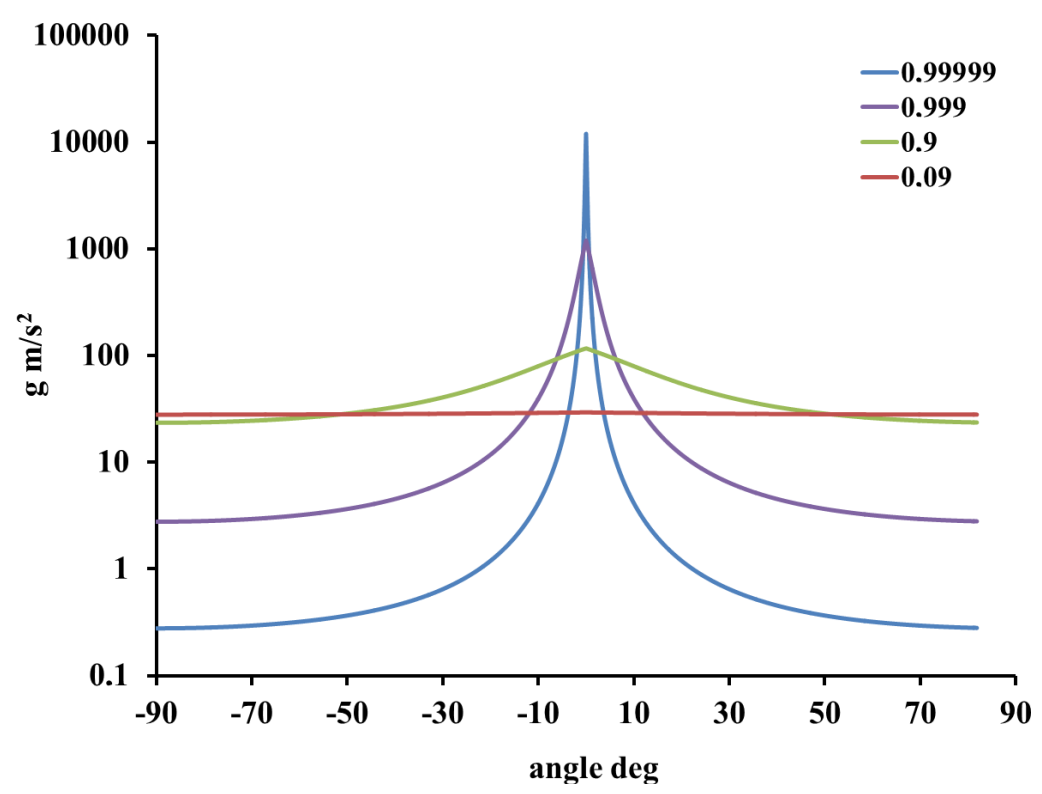

Figure 5. This show the calculated surface g for a rotating star as function of Angle, where 0 degrees is in the rotation direction (within the disc) as observed by a stationary observer (not rotating). The star is of mass $\mathrm{M}=10^{5} \mathrm{Msol}$ (measured in the rotating frame) and radius $\mathrm{R}=10^{12} \mathrm{~m}$ (measured in a stationary rest frame). The star is modeled with a constant rotation velocity of; $99.999 \%, 99.9 \%, 0.9 \%$ and $0.1 \%$ of c, corresponding to a gamma value shown in the caption box. Several orders of magnitude enhancement is seen in $g$ within the disc, in the non-disc (axial) direction several orders of magnitude reduction is seen in $\mathrm{g}$. 


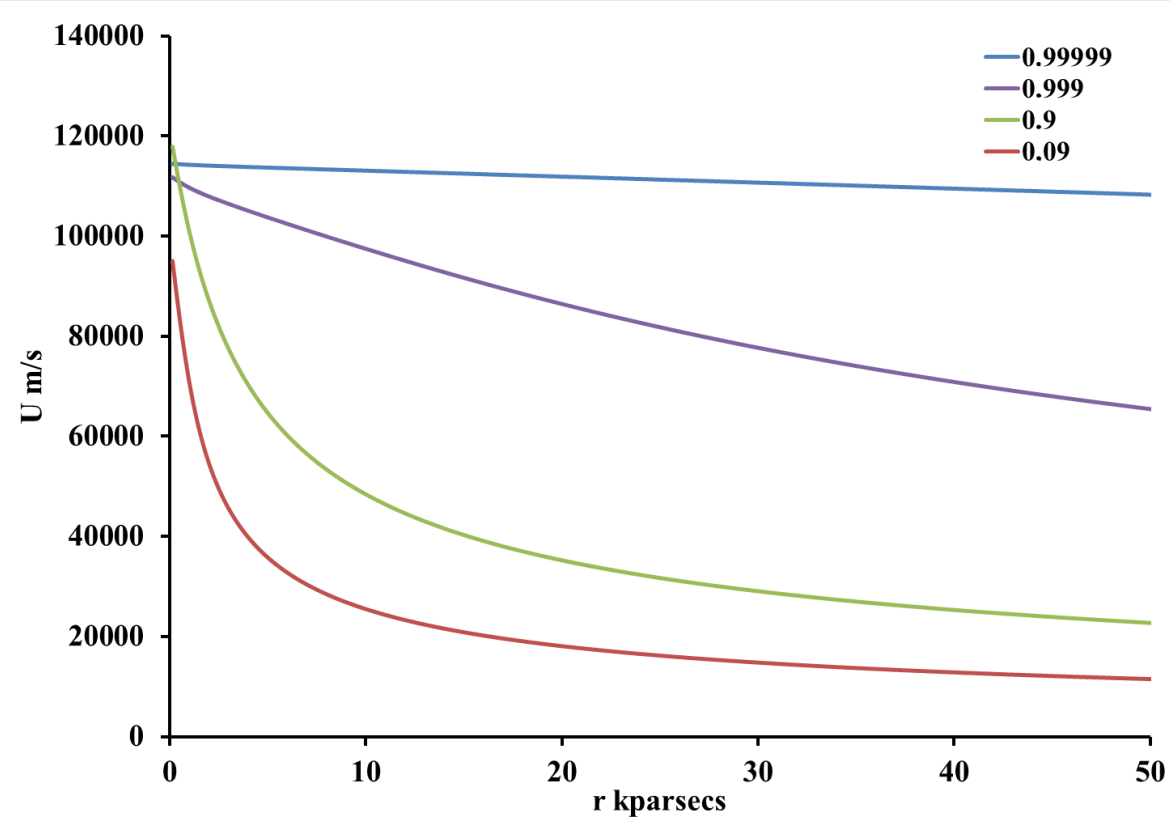

Figure 6. This shows the Galactic rotation velocity as a function of radius for a central star with rotation velocity $99.999 \%$, $99.9 \%, 0.9 \%$ and $0.1 \%$ of c, corresponding to gamma shown in caption box. The central star mass is $\mathrm{M}=10^{5} \mathrm{Msol}$ (measured in rotating frame) and radius $\mathrm{R}=10^{12} \mathrm{~m}$ (measured in stationary rest frame). The galactic disc thickness is $2 \mathrm{kpc}$ (i.e. $\mathrm{g}$ is averaged over $2 \mathrm{kpc}$ ).

observed as a non-Keplerian space-time variation i.e. that averaging over a fixed area around the rotation plane (angle $=0$ ) leads to a 1/r dependence. Again in Figure 6 a few different rotation velocities have been taken. The results show that at a rotation velocity of $99.999 \%$ of c the predicted galactic rotation curve produced from the simplified central massive star quantitatively reproduces the observed and expected approximately constant around $120 \mathrm{~km} / \mathrm{s}$ rotation velocity. In this model this value is dependent upon the star mass and rotation velocity only and not sensitive to the star radius, increasing the mass would increase the rotation velocity (as $\sqrt{M}$ ). Varying the stars radial diameter will increase the surface gravity, but not t hat measured within the disc. Decreasing the assumed disc thickness will increase the average gravitational field.

Note that the rotation velocity curve shown in Figure 6 is based upon the gravitational acceleration (within the disc) generated only from this central massive rotating star and does not include that produced by all other visible forms of mass e.g. stars, gas and dust or even mass within the star which is rotating at different velocities. The gravitational field generated by this additional distribution of mass should be included and may help explain the details of the galactic rotation velocity (Rubin et al. 1980). Note also that in this simple model if the disc thickness decreases with radius (distance from the galactic center) then this would also lead to an increasing predicted average rotation velocity with distance. There are several other potentially important factors which have not been fully implemented in this model, see Appendix 1.

Firstly precession of the star would affect the angular distribution of gravitation. Even a precession as low as 3 degrees could reduce the peak gravitational field by more than an order of magnitude. This is illustrated in Figure 7 and Figure 8 where for the same star modelled in Figure 5 and Figure 6 have included an additional integration loop due to precession of the rotation axis (of 3 degrees). As can be seen this results in a broadening in the angular distribution and structure appearing (Figure 7). This leads to a predicted rather sudden fall-off in rotation velocity at a particular radial distance $(\mathrm{d}>\mathrm{h} / \tan (\lambda))$ see Figure 8, which will depend upon the disc thickness, the precession angle $(\lambda)$ as well as the star rotation velocity.

Secondly in this simplified model non-linear effects have been ignored which in principle could be significant at these high gravitational fields. This effect will depend upon the geometry (radius) of the star and will be a second order effect compared to that of the Doppler blue shift in g. At a high enough surface gravitational field it would impose a maximum field intensity to Figure 5, though in this case it is significantly less than this limit (which can be estimated to be around $\mathrm{g}_{\text {surf }}<\mathrm{c}^{2} / \mathrm{R}=10^{5} \mathrm{~m} / \mathrm{s}^{2}$ ).

Thirdly depending upon the structure and (radial) velocity profile the geometry of the star might be expected 


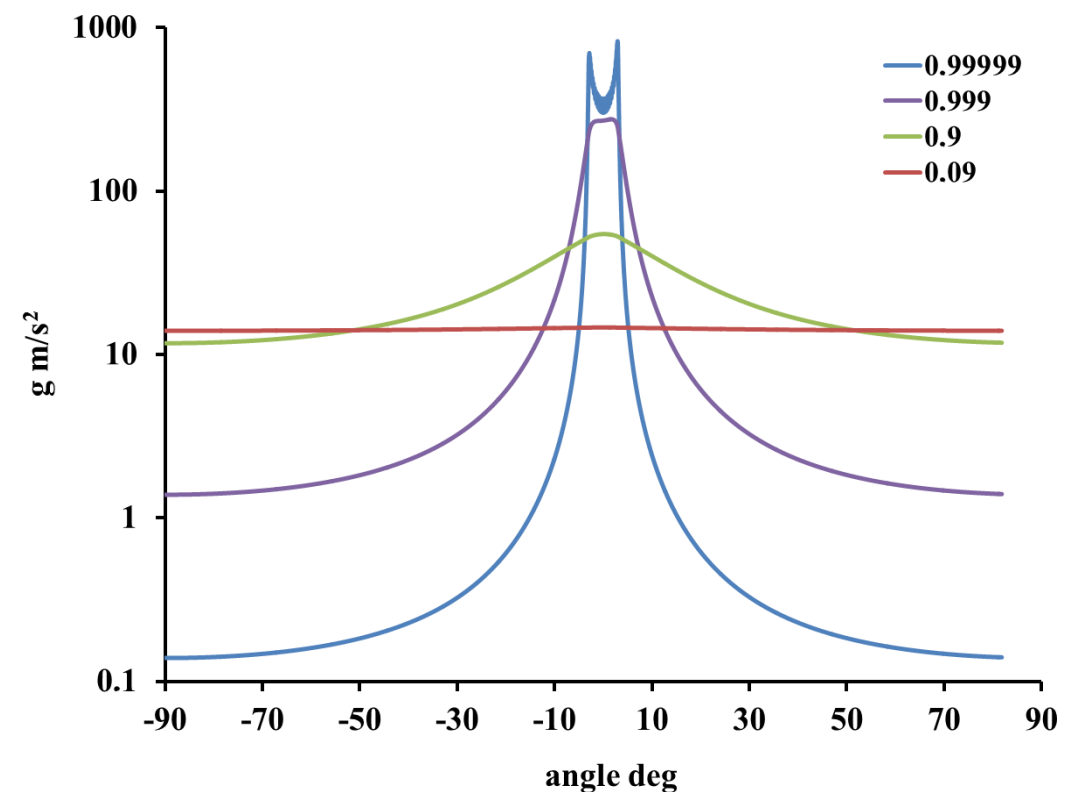

Figure 7. This shows the calculated surface $\mathrm{g}$ as a function of Angle, 0 degrees is in the rotation direction (disc) as observed by a stationary observer (not rotating). The star is precessing $\lambda=3^{\circ}$. The mass is $\mathrm{M}=10^{5}$ Msol and radius $\mathrm{R}=10^{12} \mathrm{~m}$, also with constant rotation velocity of; $99.999 \%, 99.9 \%, 0.9 \%$ and $0.1 \%$ of c, corresponding to a gamma value shown in the caption box.

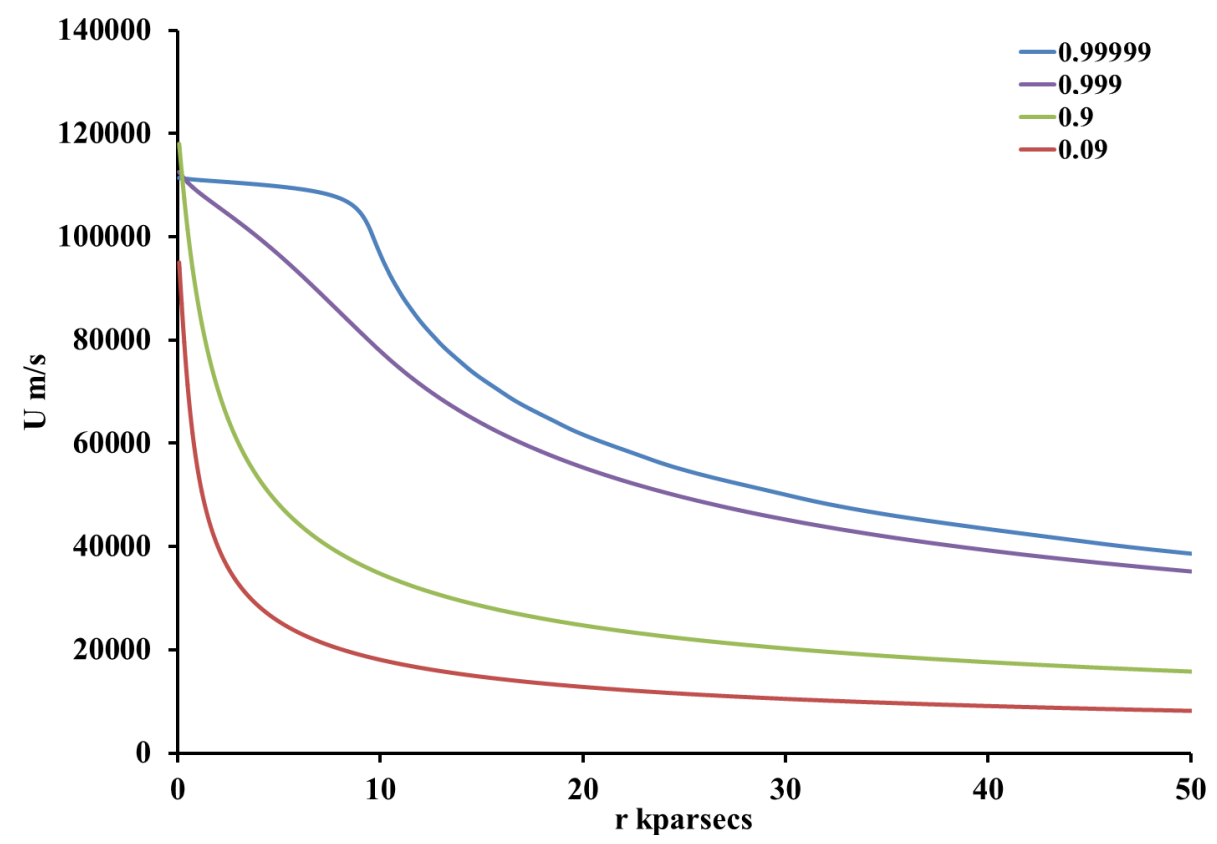

Figure 8. This shows the Galactic rotation velocity as a function of radius for a central star with rotation velocity $99.999 \%$, $99.9 \%, 0.9 \%$ and $0.1 \%$ of c, corresponding to gamma shown in caption box. The central star mass is $\mathrm{M}=10^{5} \mathrm{Msol}$ (measured in rotating frame) and radius $\mathrm{R}=10^{12} \mathrm{~m}$ (measured in stationary rest frame). The star is precessing $\lambda=3^{\circ}$. The galactic disc thickness is assumed to be $1 \mathrm{kpc}$ (i.e. $\mathrm{g}$ is averaged over $1 \mathrm{kpc}$ ).

to be non-spherical. In reality there will be various rotational velocities within the star and possibly also other rapidly rotating stars within the central region. This may help to explain the complex structure and variation in galactic rotation velocities. The possible effects of magnetic fields have not been considered here, but may also contribute.

Several features expected for a rapidly rotating massive star are qualitatively observed in the central regions 
of galaxies (e.g. in active galactic nuclei). As shown in Figure 5 this model, as well as enhancing the gravitational field in the plane of rotation (angle $\rightarrow 0$ ), the gravitational field is dramatically reduced in the axial direction (angle $\rightarrow 90$ ). Such a star might therefore be expected to generate axial jets again qualitatively agreeing with some observations. Since any matter encountering the high velocity surface will generate large amounts of electromagnetic energy the star may also be expected to be a source of electromagnetism (with high Doppler shifts) and also emit high energy particle. The large angular variation in gravitational field around the star would probably lead to complex non-Keplerain orbits and possibly also general instability and/or heating. The highly anisotropic gravitational field predicted to surround a rapidly rotating and precessing star in this model would also be expected to lead to high temporal variability (possibly several orders of magnitude)in observable light output in several ways; 1) through Doppler shift in thermal radiation, 2) through in-falling matter encountering the surface with the rate due to the gravitational field, 3) circumstellar material (gas) light output should be related to the temperature, pressure and therefore gravity; $T(t) \propto P(t) \propto g(t)$.

For the Milky-way galaxy a possible candidate for this star might be Saggitarius. $A^{*}$. The reasoning here is that it generates high gravitation, it is a radio and x-ray source, it is close to central. However, the existence of stable orbiting stars close to Saggitarius. A* (e.g. S2, S1, S14, etc.) seems not to support this as a viable choice for this central highly rotating object. Unfortunately the simple model presented here breaks down at radial distances comparable to the disc thickness and a more sophisticated approach is required for the inner galactic region. It is beyond the scope of this study to conclude whether Saggitarius A* is a likely candidate for such a rapidly rotating massive star or not. This would require detailed modelling and comparison with astronomical observations.

Clusters of gravitationally bound galaxies are observed which cannot be easily explained by conventional models of gravity (without the need to assume dark matter). In this model the gravitational 'sheet' produced by $\mathrm{aM}=10^{5}$ Msol star with rotation velocity $99.999 \% \mathrm{c}$ and radius $\mathrm{R}=10^{12} \mathrm{~m}$ has been calculated (see Figure 9). This is averaged over a $2 \mathrm{kpc}$ thick section around the rotation plane and extended to a radial distance of around $1000 \mathrm{kpc}$. At this distance the rotation velocity is predicted to be around $100 \mathrm{~km} / \mathrm{s}$ agreeing crudely with values observed for velocities within gravitationally bound galaxy clusters [15]. This type of gravitational 'sheet' may also help explain the binding of galactic super-clusters into sheet (filament) type structures [23].

\section{Solar System Tests}

Within the solar system it is possible to perform high precision tests of general relativity, typically studying pla-

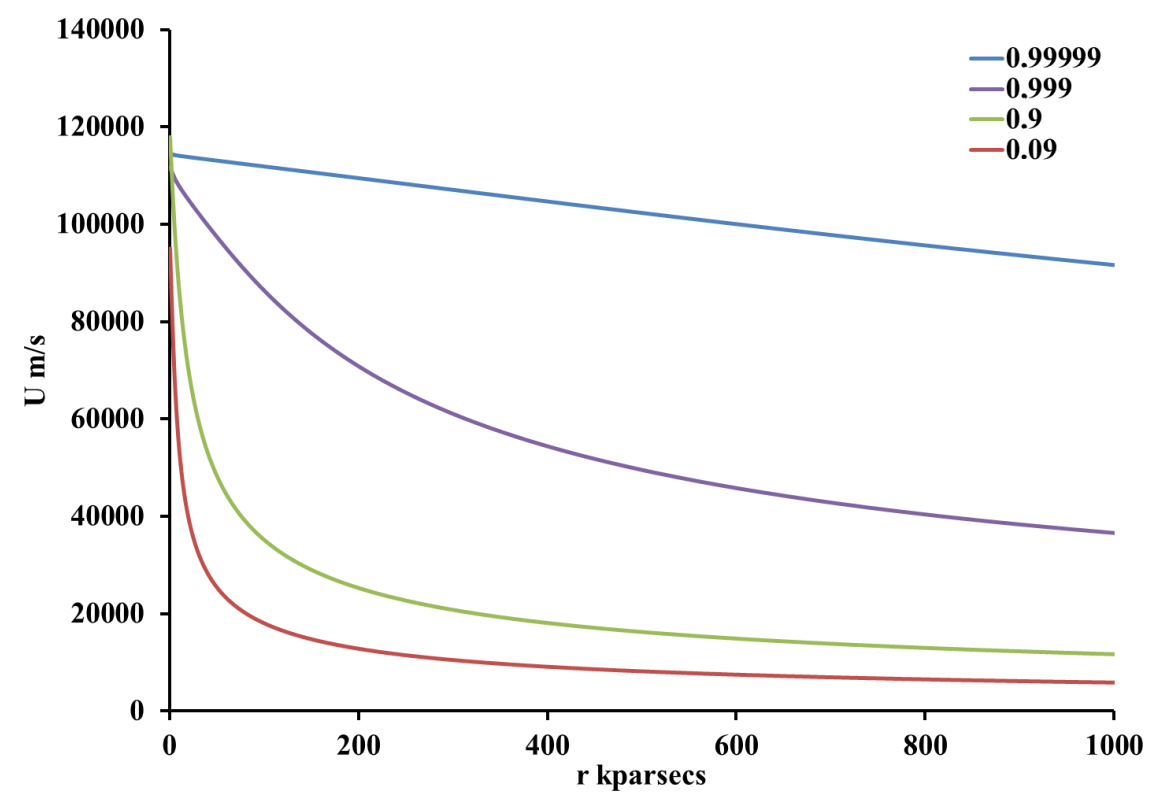

Figure 9. This shows the Galactic rotation velocity as a function of radius for a central star with rotation velocity $99.999 \%$, $99.9 \%, 0.9 \%$ and $0.1 \%$ of c, corresponding to gamma shown in caption box. The central star mass is $\mathrm{M}=10^{5} \mathrm{Msol}$ (measured in rotating frame) and radius $\mathrm{R}=10^{12} \mathrm{~m}$ (measured in stationary rest frame). The galactic discthickness is $2 \mathrm{kpc}$ (i.e. $\mathrm{g}$ is averaged over $2 \mathrm{kpc}$ ) and there is no precession. This is similar to Figure 6 except the range is extended to $1000 \mathrm{kpc}$. 
netary/satellite orbits and also performing tests involving gravitationally induced time dilation. It is also possible to test aspects of equivalence. The success of general relativity is in large part due to its success in such tests. These effects of (gravitationally induced) time dilation and electromagnetic field propagation in general relativity will not be strongly affected by this new model which only treats (modifies) the propagation of gravitational fields.

Another way of re-stating the model presented here is that general relativity as it is applied conventionally is a weak field approximation which accounts for time dilation effects and propagation effects of electromagnetic energy-momentum which are not significantly affected in this model the focus here is on the propagation effects of the gravitational field. Similarly the equivalence of inertial and gravitational mass is assumed here (the weak equivalence principle). However the assumption of an accelerating (accelerated) reference frame being indistinguishable from a rest frame is only valid in a weak field approximation, but not a viable assumption (especially at high field and high velocity).

The corrections suggested here for general relativity include specifically; a Doppler shift (relative velocity) and non-linearity due to gravity-gravity interaction. At high velocity (section 3) and high field/large distance (section 2) these effects are extremely significant. However in the weak field/low velocity seen in the solar system the effects are small and difficult to isolate [22] [24]. The aim in this section is to perform some crude rough order of magnitude (ROM) calculations to estimate the magnitude of these corrections to parameters within the solar system and compare them to observations. This is not for the purpose of verifying this model, merely in order to illustrate that definitive proof against this model appears to be lacking.

Gravitational non-linear effects can be crudely quantified for the orbits of solar system planets by estimating $\mathrm{dg} / \mathrm{g}=\mathrm{gdr} / \mathrm{c}^{2}$, using $\mathrm{dr} \sim 10^{12} \mathrm{~m}$ and $\mathrm{g} \sim 10^{-4} \mathrm{~m} / \mathrm{s}^{2}$ this is of the order $\mathrm{dg} / \mathrm{g} \sim 10^{-9}$ or $\mathrm{dg}=10^{-13} \mathrm{~m} / \mathrm{s}^{2}$. This is a high precision. This effect could also be seen as a variation in $\mathrm{G}$, i.e. a deviation from $\mathrm{GM} / \mathrm{r}^{2}$ or a gradient in $\mathrm{G}$ within the solar system, however the accuracy of direct (laboratory and satellite) determinations here are dG/G around $10^{-4}[24]$.

A more stringent test of this model could be the observed velocity induced dependence in $g\left(g_{\text {Doppler }}\right)$. A prediction of this model which could in principle be experimentally verified is that an object moving towards a gravitating body, for example a planet or satellite, would experience a blue shifted (enhanced) gravitation compared to one travelling away from the body i.e. an object moving upwards would experience $g /(\gamma(1+v / c))$, downwards $g /(\gamma(1-v / c))$ or horizontally $(\gamma g)$. For such an experiment at the surface of the Earth would require a precision of the order v/c which is extremely challenging, requiring either high velocity and/or high precision. Several other possible solar system probes of this effect will be discussed here, specifically satellite trajectories e.g. flybys, planetary rotation and planetary orbits.

Flyby

In order to probe the Doppler induced shift in gravity a satellite with a relative mean velocity in the direction of a massive object (planet) of for example $v_{\text {rad }}=2 \times 10^{3} \mathrm{~m} / \mathrm{s}$ would require a precision in measuring the acceleration of $1 /(\gamma(1-v / c)) \sim\left(v_{\text {rad }} / c\right)<10^{-5}$. In fact there are several observed (so called) flyby anomalies. An ROM of this effect is around; $\mathrm{d} v_{\text {Dopp }}=\left(v_{\text {rad }}\right)^{2} / c=13 \mathrm{~mm} / \mathrm{s}$, where $\mathrm{d} v_{\text {Dopp }}$ is the deviation in velocity attained during the flyby compared to that expected conventionally. This is the same order of magnitude of some observed flyby anomalies (several $\mathrm{mm} / \mathrm{s}$ ).

Planetary rotation

For the rotation of planets (in our solar system) one crude method of evaluating the Doppler effect in gravitational propagation is to compare the magnitude of the change in gravitational acceleration with that of the 'tidal' gradient i.e. the decrease due to the spatial variation in solar gravitational field. For the case of the Doppler shift for both Earth and Jupiter a crude approximation shows them be of the order of;

$$
g_{\text {Doppler }} \approx\left(v_{\text {rot }} / c\right) g_{\text {sol }} \approx 9 \times 10^{-9} \mathrm{~m} / \mathrm{s}^{2}
$$

This can be compared to respectively the tidal gradients across the planets given by;

$$
g_{\text {Tidal }} \approx 2 R_{\text {planet }} G M_{\text {sol }} / r_{\text {sol }}{ }^{3}
$$

Thus giving; Earth; $g_{\text {Tidal }} \approx 5 \times 10^{-7} \mathrm{~m} / \mathrm{s}^{2}$ and Jupiter; $g_{\text {Tidal }} \approx 4 \times 10^{-8} \mathrm{~m} / \mathrm{s}^{2}$.

The gravitational Doppler effect is therefore comparable, but significantly smaller than the tidal gradient. The Doppler effect in gravity is unlike the tidal effect in that it will seek to accelerate the rotational velocity instead 
of reducing it. It may therefore contribute a small additional source of internal heating. This effect would be difficult to isolate observationally given uncertainties in planetary internal structure and dynamics.

\section{Planetary orbits}

In the case of the orbits of planets the rotation of the sun would be expected (through a gravitational Doppler effect) to lead to a systematic shift in the center of gravity and therefore a shift in the gravitational barycenter. This could lead to a retrograde (with respect to the rotation of the sun) precession of the planets orbit. In the case of Mercury this would be in addition to, for example, the effect of other planets which corresponds to around 532"/century i.e. $\left(\frac{\omega_{\text {prec }}}{\omega_{\text {orbital }}}\right)_{\text {mercury }} \approx 10^{-6}$ radians/radian and the effect of Mercury's slight elliptical orbit as (successfully) predicted by general relativity of around 43"/century i.e. $\left(\frac{\omega_{\text {prec }}}{\omega_{\text {orbital }}}\right)_{\text {mercury }} \approx 8 \times 10^{-8}$.

A crude quantification of this effect can be made by estimating the average radial velocity component in the planets direction (for the entire sun). This was determined by numerically (computationally) integrating the velocity and mass within the sun in order to determine the average speed in the direction of Mercury. The internal mass distribution of the sun was assumed based upon the 'standard model', specifically a mass density distribution of;

$$
D(x)=519 x^{4}-1630 x^{3}+1844 x^{2}-889 x+155 \mathrm{~g} / \mathrm{cm}^{3},
$$

where $x$ is the relative radial position i.e. $x=\mathrm{r} / \mathrm{Rsol}$, (see [25]), the rotational angular velocity of the sun was taken as $430 \mathrm{nHz}$ [26]. This numerical integration yielded a value of around; $U_{a v} \approx 60 \mathrm{~m} / \mathrm{s}$ leading to a shift in the barycenter of around $\Delta r \approx\left(\frac{U_{a v}}{c}\right) R_{s o l}$.

$$
\frac{\omega_{\text {prec }}}{\omega_{\text {orbit }}} \approx \frac{\Delta r}{r_{\text {sol }}} \approx \frac{U_{a v}}{C} \cdot \frac{R_{\text {sol }}}{r_{\text {sol }}}
$$

where $R_{\text {sol }}$ is the distance to the sun, $R_{\text {sol }}$ is the radius of the sun and $\frac{\omega_{\text {prec }}}{\omega_{\text {orbit }}}$ is the ratio between the angular velocity of precession to that of the orbital (also the ratio in precession and orbital periods). For the case of Mercury $\Delta r$ is around $140 \mathrm{~m}$ (compared to around $9000 \mathrm{~m}$ offset of Mercuries barycenter compared to the center of the sun), this then gives a predicted (ROM) perihelion (apsidal) precession of; $\frac{\omega_{\text {prec }}}{\omega_{\text {orbit }}} \approx 2.4 \times 10^{-9}$ or 1.3 "/century. This is comparable to the estimated observational uncertainty i.e. around 0.65 "/century or $\frac{\omega_{\text {prec }}}{\omega_{\text {orbit }}}$ $\approx 1.2 \times 10^{-9}$ (e.g. [27]) and small compared to other contributions. As can be seen the precession due to Doppler shifting of gravity would be expected for other planets to decrease with distance from the sun as $\left(1 / R_{\text {sol }}\right)$.

In summary several potential tests of this model have been investigated using (ROM) estimates e.g. of; planetary precessions, flyby anomalies, spacecraft anomaly. They seem not to be in obvious disagreement with observations at the level of accuracy of these estimates [24]. More stringent tests of this model should be performed.

\section{Conclusions}

In this study a model is proposed in which general relativity is modified by propagating the gravitational field in an analogous manner to that of an electromagnetic field. Specifically this entails the inclusion of velocity induced changes in gravitational field propagation (relativistic Doppler effects) and non-linearity (the effect of gravity upon itself in vacuum). In the work presented here a simple computational approach has been taken to quantify these effects.

The inclusion of a Doppler shift in gravitational fields allow the rotation velocities of galaxies to be quantitatively reproduced simply by assuming a central rapidly rotating, moderately high mass $\left(<10^{6} \mathrm{Msol}\right)$ star. This also (quantitatively) explains the gravitational coupling of galaxies within clusters and supports observations of 
non-Keplerian (non-Newtonian) motion for gravitational systems on large scales. This results from the highly anisotropic gravitational field emitted at high (relativistic) rotation velocities. To re-iterate in this model it is suggested here that a massive extremely rapidly rotating star can generate an intense sheet of gravitation and thereby explain the almost constant rotation velocity component observed in many galaxies. A similarly (or even much greater) mass star at rest would not produce such an effect. The many varied forms of galaxies may reflect the distribution in spin velocity of the central massive star or even be due to a combination of more than one such high velocity rotating star.

The non-linearity resulting from the self-interaction of the gravitation field (in vacuum) has several important consequences. It prevents the anomalous and paradoxical generation of infinite space-time curvature (singularity) or infinite gravitationally-generated red-shift (an event horizon) i.e. black holes will not be possible. In this model the gravitational field will always be real and finite since a red-shift in the gravitational field prevents reaching the point at which light (or gravitational field) cannot escape. Increasing the density of a massive object will ultimately lead to reduction in the gravitational field compared to that expected under general relativity (or Newtonian gravitation). In this case there becomes an effective 'penetration' depth of gravity due to its selfcoupling. This depth is crudely around; $d \sim c / \sqrt{4 \rho G}$.

In this model a specific 'causality' of gravitation is made analogous to electro-magnetism, i.e. the assumption (and one which is central to relativity theory) is that gravitational energy exchange is analogous to that of a photon. This allows gravity to be compatible with other field theories and unified with all other forces i.e. compatible with unified theories and/or quantum theories. The work here imposes few restrictions on the form of the carrier of gravitational information other than it resembles a photon/electromagnetic field perturbation.

Although this model is not a purely geometric model of gravitation, general relativity is assumed to be an accurate approximation at low fields and low velocities, specifically where time dilation and/or transport of electromagnetic fields are the dominant factors. Therefore many precision tests of general relativity within the solar system are also valid in this model. However this model proposes that gravitational fields must be propagated in the same way as electromagnetic fields and therefore in addition to time dilation effects and those of electromagnetic propagation, the propagation of gravity fields must also be included. Typically within the solar system these effects are small compared to measurement precision and/or other dominant physical processes. It seems that there is no definitive evidence to disprove the model proposed here. It is hoped that this work stimulates further experimental/observational tests of gravitational models, specifically more detailed calculation using more extensive computing power.

\section{References}

[1] Rubin, V.C., Ford, W.K. and Thonnard, N. (1980) Rotational Properties of 21 Sc Galaxies with a Large Range of Luminosities and Radii, from NGC 4605 ( $\mathrm{R}=4 \mathrm{kpc}$ ) to UGC 2885 (R = $122 \mathrm{kpc}$ ). The Astronomical Journal, 238, 471-487. http://dx.doi.org/10.1086/158003

[2] Brownstein, J.R. and Moffat, J.W. (2007) The Bullet Cluster 1E0657-558 Evidence Shows Modified Gravity in the Absence of Dark Matter. Monthly Notices of the Royal Astronomical Society, 382, 29-47. http://dx.doi.org/10.1111/j.1365-2966.2007.12275.x

[3] Sanders, R.H. (1990) Mass Discrepancies in Galaxies: Dark Matter and Alternatives. The Astronomy and Astrophysics Review, 2, 1. http://dx.doi.org/10.1007/BF00873540

[4] Bugg, D.V. (2013) A New Perspective on MOND. Canadian Journal of Physics, 91, 668-675. http://dx.doi.org/10.1139/cjp-2013-0163

[5] Mendoza, S. and Gonzalo, J.O. (2015) Astrophysical Constraints and Insights on Extended Relativistic Gravity. Astrophysics and Space Science, 357, 133. http://dx.doi.org/10.1007/s10509-015-2363-y

[6] Famaey, B. and McGaugh, S.S. (2012) Modified Newtonian Dynamics (MOND): Observational Phenomenology and Relativistic Extensions. Living Reviews in Relativity, 15, 10. http://dx.doi.org/10.12942/lrr-2012-10

[7] Hernandez, X., Jiménez, M.A. and Allen, C. (2012) Wide Binaries as a Critical Test of Classical Gravity. The European Physical Journal C, 72, 1884. http://dx.doi.org/10.1140/epjc/s10052-012-1884-6

[8] Hernandez, X. and Jiménez, M.A. (2012) The Outskirts of Globular Clusters as Modified Gravity Probes. The Astrophysical Journal, 750, 1-9. http://dx.doi.org/10.1088/0004-637X/750/1/9

[9] Mendoza, S., Bernal, T., Hernandez, X., Hidalgo, J.C. and Torres, L.A. (2013) Gravitational Lensing with $f(\chi)=\chi^{3 / 2}$ Gravity in Accordance with Astrophysical Observations. Monthly Notices of the Royal Astronomical Society, 433, 
1802-1812. http://dx.doi.org/10.1093/mnras/stt752

[10] Reyes, R., et al. (2010) Confirmation of General Relativity on Large Scales from Weak Lensing and Galaxy Velocities. Nature, 464, 256-258. http://dx.doi.org/10.1038/nature08857

[11] Peebles, P. (1980) The Large Scale Structure of the Universe. Princeton University Press, Princeton.

[12] Evans, N.W. (1996) The Nature of the Galactic Dark Matter. Monthly Notices of the Royal Astronomical Society, 278, L5-L9. http://dx.doi.org/10.1093/mnras/278.1.L5

[13] Milgrom, M. (1983) A Modification of the Newtonian Dynamics as a Possible Alternative to the Hidden Mass Hypothesis. The Astrophysical Journal, 270, 365-370. http://dx.doi.org/10.1086/161130

[14] Milgrom, M. (2014) MOND Laws of Galactic Dynamics. Monthly Notices of the Royal Astronomical Society, 437, 2531-2541. http://dx.doi.org/10.1093/mnras/stt2066

[15] Mould, J., Colless, M., Erdoğdu, P., Jones, H., Lucey, J., Ma, Y., Magoulas, C. and Springob, C.M. (2015) Modified Gravity and Large Scale Flows. Astrophysics and Space Science, 357, 162. http://dx.doi.org/10.1007/s10509-015-2351-2

[16] Sanders, R. (2015) A Historical Perspective on Modified Newtonian Dynamics. Canadian Journal of Physics, 93, 126-138. http://dx.doi.org/10.1139/cjp-2014-0206

[17] Bothun, G. (2015) The Motion of Test Particles and Cosmological Interpretations: The Role of MOND. Canadian Journal of Physics, 93, 139-150. http://dx.doi.org/10.1139/cjp-2014-0165

[18] Bekenstein, J. (2004) Relativistic Gravitation Theory for the Modified Newtonian Dynamics Paradigm. Physical Review D, 70, Article ID: 083509. http://dx.doi.org/10.1103/PhysRevD.70.083509

[19] Moffat, J.W. and Toth, V.T. (2015) Rotational Velocity Curves in the Milky Way as a Test of Modified Gravity. Physical Review D, 91, Article ID: 043004. http://dx.doi.org/10.1103/PhysRevD.91.043004

[20] Moffat, J.W. and Rahvar, S. (2013) The MOG Weak Field Approximation and Observational Test of Galaxy Rotation Curves. Monthly Notices of the Royal Astronomical Society, 436, 1439-1451. http://dx.doi.org/10.1093/mnras/stt1670

[21] Clifton, T., Ferreira, P.G., Padilla, A. and Skordis, C. (2012) Modified Gravity and Cosmology. Physics Reports, 513, 1-189. http://dx.doi.org/10.1016/j.physrep.2012.01.001

[22] Will, C.M. (2014) The Confrontation between General Relativity and Experiment. Living Reviews in Relativity, 17, 4. http://dx.doi.org/10.12942/lrr-2014-4

[23] Tully, R.B., Courtois, H., Hoffman, Y. and Pomarède, D. (2014) The Laniakea Supercluster of Galaxies. Nature, 513, 71-73. http://dx.doi.org/10.1038/nature13674

[24] Turyshev, S.G. (2009) Experimental Tests of General Relativity: Recent Progress and Future Directions. Physics-Uspekhi, 52, 1-27. http://dx.doi.org/10.3367/UFNe.0179.200901a.0003

[25] http://spacemath.gsfc.nasa.gov/weekly/6Page102.pdf

[26] Christensen-Dalsgaard, J. and Thompson, M.J. (2007) The Solar Tachocline Observational Results and Issues Concerning the Tachocline. Cambridge University Press, Cambridge, 53-86.

[27] Clemence, G.M. (1947) The Relativity Effect in Planetary Motions. Reviews of Modern Physics, 19, 361-364. http://dx.doi.org/10.1103/RevModPhys.19.361 


\section{Appendix 1}

Equation (1) (section 2) is the basis for each of the computational calculations, i.e. the observed gravitational field in a frame of reference having a small velocity shift relative to the adjacent frame.

In the case of the calculation in section 2 the effect of the gravitational field upon its own propagation is included.

In the case of calculation in section 3 the velocity of the gravitating mass relative to the observer is included.

\section{Computation 1 (section 2)}

The model assumes a gravitating, spherical, symmetric, non-rotating gravitating mass with a radial $(r)$ mass distribution $M(r)=M \exp (-r / h)$ to a maximum radius rmax of from $10^{-7}$ Rsol to 1 Rsol and scale height $\mathrm{h}=$ $\operatorname{rmax} / 8$, the total Mass varied between $1 \mathrm{Msol}$ and $10^{7}$ Msol.

The first iteration of the gravitational field is based upon the Newtonian gravitational field.

The goal of the calculation is to obtain a new gravitational field $g(r)$ which is stable against repeated propagation i.e. differs little from the previous iteration when propagating the gravitational field. Field propagation consists of;

- Integration over discrete mass elements at different radius; $r=0$ to rmax.

- Integration over emission angle with respect to the axial direction (polar angle)

- Integration over emission angle with respect to the radial direction (azimuthal angle)

- Integration over time as the gravitation field is propagated (at $c$ ) from frame to frame.

This constitutes four nested loops plus repeated iterations.

\section{Computation 2 (section 3)}

This model assumes constant rotational velocity $(v)$ of the gravitating mass, this is made only for simplicity.

The gravitational field at radius $r$ from each gravitating mass element $g(r)$ is computed.

- Integration over emitting angle is made i.e. integration over azimuthal angle i.e. around the rotating axis.

- Averaging is made over the thickness of the galactic disc, this contributes an additional angle to that of the rotation plane.

This consists of two nested loops.

Note that clearly one side of the star is rotating towards the observer and has a blue shift whereas the other side of the star is rotating away from the observer and has a redshift.

\section{Submit or recommend next manuscript to SCIRP and we will provide best service for you:}

Accepting pre-submission inquiries through Email, Facebook, LinkedIn, Twitter, etc.

A wide selection of journals (inclusive of 9 subjects, more than 200 journals)

Providing 24-hour high-quality service

User-friendly online submission system

Fair and swift peer-review system

Efficient typesetting and proofreading procedure

Display of the result of downloads and visits, as well as the number of cited articles

Maximum dissemination of your research work

Submit your manuscript at: http://papersubmission.scirp.org/

Or contactijaa@scirp.org 